\title{
The Growth Kinetics of Sucrose in Presence of Glucose in an Isothermal Continuous-Flow Reactor using a Photomicroscopic Method
}

\author{
L. D. Shiau
}

Dept. of Chemical and Materials Engineering, Chang Gung University, Taoyuan, Taiwan R.O.C

The photomicroscopic technique in an isothermal, continuous-flow growth chamber is employed in this work to study the growth kinetics of the contact nuclei in the aqueous sucrose system. In a continuousflow growth chamber, the resistance to crystal growth due to the volume diffusion may be reduced or even eliminated by increasing the solution flow rate through the growth chamber. Therefore, the effects of the sole surface reaction step on the growth kinetics can be investigated [1]. In addition, the effects of the sole surface reaction step on the growth kinetics of sucrose due to the presence of glucose will be studied.

A schematic diagram of the cell with a description of features is shown in Fig. 1 [2]. The cell has a chamber for crystal nucleation and growth in the upper part and a chamber for temperature-controlled water in the lower part. A model will be presented to explain the observed growth phenomenon. An effective factor is introduced to take into account the growth promoting ability of impurity adsorbed on the crystal surface. The linear growth rate in the presence of impurities is proposed as

$$
G=G_{0}[(1-\theta)+\alpha \theta]
$$

Where $G_{0}$ is the linear growth rate in the pure system, $\theta$ is the fractional coverage by adsorbed impurity on the crystal surface and $\alpha$ is the effective factor to take into account the growth promoting or retarding ability of the impurity adsorbed on the crystal surface.

As described earlier, the diffusion process proceeds very fast because the flow velocity is high in this work. If the adsorption process of impurity on the crystal surface proceeds very fast, the fractional coverage, $\theta$, is replaced by equilibrium value, $\theta_{\text {eq }}$, and Langmuir adsorption isotherm can be applied. That is,

$$
\theta_{e q}=K x /(1+K x)
$$

Where $K$ is the Langmuir constant and $x$ is the impurity concentration in solution. From Eqs. (1) and (2), we have

$$
G / G_{0}=(1+\alpha K x) /(1+K x)
$$

Since $\alpha$ is inversely proportional to the supersaturation of sucrose in the experimental data, $\alpha$ is replace by $a / \sigma$ for simplicity. Thus, Eq.(3) can be written as

$$
G / G_{0}=(1+a K x / \sigma) /(1+K x)
$$


Parameters $K$ and $a$ can thus be determined by the nonlinear regression using the results of experiments. Fig. 2 depicts the relative growth rate $G / G_{0}$ as a function of impurity mole ratio $x$ for three levels of supersaturation. The experimental data are found to be satisfactorily fitted well by the proposed model in Eq.(4).

References:

[1] Shiau, L. D., Chem. Eng. Sci. 58 (2003), p. 5299.

[2] Shiau, L. D., Berglund, K. A., AIChE J. 33 (1987), p. 1028.
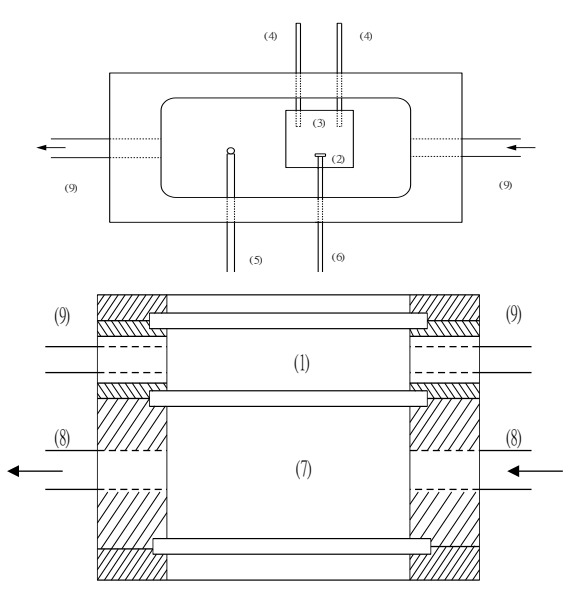

Figure 1. Schematic diagram of nucleation cell with the features (1) chamber containing solution (2) parent crystal (3) glass cover slip where parent crystal is slid (4) support rods for glass cover slip (5) thermistor (6) movable rod holding parent crystal (7) chamber containing constant temperature water (8) water inlet and outlet and (9) solution inlet and outlet.

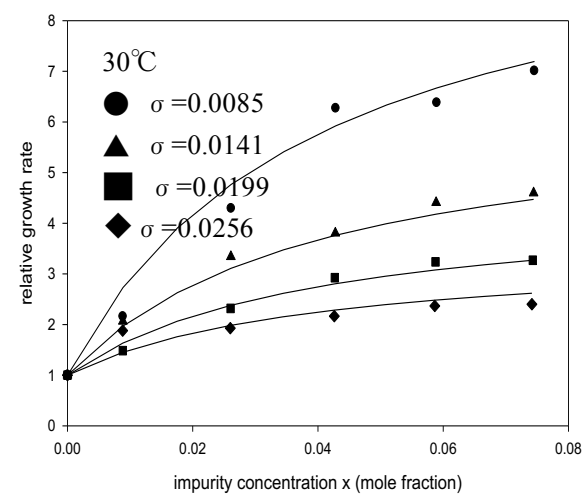

Figure 2. Relative growth rate versus impurity concentration. 